\title{
El ambiente en la agenda periodística
}

LISBETH FOG CORRADINE

\section{RESUMEN}

El ambiente en la agenda periodística da cuenta de las dinámicas y de las relaciones entre los hechos que son noticia en el campo ambiental, los medios de comunicación y la ciudadanía. Inicia con una mirada a las páginas de medio ambiente en los diarios colombianos durante tres meses del 2006, hace un recuento de los diferentes enfoques de los medios masivos de comunicación frente al tema ambiental, para concentrarse luego en las historias periodísticas sobre ambiente, desde la generación de la idea hasta el impacto que tiene sobre el público receptor. Este proceso realiza tambien una mirada general a la investigación científica en Colombia, en especial, la que se realiza en temas ambientales y en particular en el departamento de Córdoba.

\section{PALABRAS CLAVE}

Periodismo ambiental - ambiente
En un país tan biodiverso como Colombia, es difícil para un periodista olvidar que el tema ambiental es parte de su agenda editorial. Sin embargo, de todos los diarios que circulan en el país, sólo tres mantenían en el 2006, una página periódica en temas ambientales: Vanguardia Liberal, de Bucaramanga, con la página titulada Ola Verde, El Nuevo Día, de Ibagué, con su página Ecológica y El Colombiano, de Medellín, con su página Medio Ambiente. Periódicos como La Patria, El Heraldo, y El Espectador, publican esporádicamente páginas sobre ambiente. Eltiempo.com titula una sección como ciencia-ecología, en la que incluye información sobre ambiente, haciendo eco también y prioritariamente a la información que proviene de cables internacionales.

Eso no significa que los demás diarios no cubran los temas que tienen que ver con la situación ambiental del país y del mundo. Por supuesto que sí lo hacen. Basta con revisar no solamente los medios impresos, sino el cubrimiento de radio, televisión, e internet sobre el calentamiento global que en el 2007 ha llenado páginas y espacios, ha ocupado portadas y avances informativos con asiduidad.

El ambiente es y ha sido noticia por la influencia que tiene en el ser humano, en su salud y en la naturaleza donde se mueve. Así, el periodismo ambiental se ha constituido como una especialidad periodística que se centra en informar sobre las relaciones entre el individuo con su entorno, el hábitat donde se desarrolla.

\section{Breve historia del periodismo ambiental}

En 1887, Stephen Forbes se acerca al concepto de la ecología en su tratado sobre los lagos como microcosmos. Ernst Haeckel, en 1906, ya se refiere a la ecología, como la parte de la biología que estudia las interrelaciones de los seres vivos entre sí y con su medio, y en 1935, Arthur George Tansley habla del ecosistema como la unidad natural básica de la biosfera. 
Desde finales del siglo XIX hasta 1940, la atención mundial se centra en la protección de la pesca y del recurso hídrico, y continúa la tendencia de una política ambiental enfocada hacia la conservación; culminando el año 1972 se llama la atención sobre la diversidad biológica, A mediados del siglo XX, el concepto de medio ambiente relaciona el conjunto de las condiciones naturales (físicas, químicas y biológicas) con las culturales y su influencia en los organismos vivos y en las actividades humanas.

El nacimiento de Greenpeace en 1971 y la Convención de las Naciones Unidas sobre Medio Humano que concluyen con el Informe Brundtland en 1972, permiten dar un giro en el enfoque ambiental. El primero inicia actividades de denuncia, diseña y desarrolla estrategias que tradicionalmente han tenido gran impacto; y el segundo, representa un cambio en la política ambiental

\section{LISBETH FOG CORRADINE :}

Comunicadora social, periodista de la Universidad Jorge Tadeo Lozano, con maestría en reportería científica de la Universidad de Boston, EEUU. Desde 1984 divulga temas de ciencia en los medios masivos de comunicación y forma comunicadores especializados a través de cursos-talleres de periodismo científico.

Es autora de libros, directora de la serie de televisión Universos, editora, traductora, investigadora, docente, conferencista nacional e internacional, y periodista independiente de varios medios masivos de comunicación.

Becaria Fulbright (1991-1993) y presidente de la Asociación Colombiana de Periodismo Científico, ACPC (1998-2004), pertenece a grupos de trabajo internacionales sobre comunicación de la ciencia. Actualmente dirige dos programas sobre ciencia y tecnología en la Radio Nacional de Colombia. Colabora con el portal www.scidev.net, y escribe para Semana, Arcadia y El Espectador. Es docente en las universidades Externado de Colombia y del Rosario.

Para contactar a la autora: lisfog@yahoo.com global generando conciencia en los ciudadanos y en quienes toman decisiones.

A finales de la década de los ochenta se inician los preparativos para la Convención de Diversidad Biológica que tiene lugar en Río de Janeiro, Brasil, en 1992, con la participación de 178 gobiernos, 102 jefes de Estado, y alrededor de 9.000 periodistas.

A partir de 1993, los grandes temas como la desertificación, la capa de ozono, el cambio climático, la bioseguridad, la relación entre ambiente y comercio, son protagonistas globales de reuniones que promueven reglas del juego para hacer frente a situaciones que afectan la calidad de vida de los seres humanos.

Este rápido recorrido por lo que ha sido la evolución de la política ambiental internacional ${ }^{1}$ incide en la manera como la prensa cubre periodísticamente estos hechos. De acuerdo con Francisco Esteve Ramírez y Javier Fernández del Moral, la década de los años 60 se caracteriza por un enfoque proteccionista, en el que los medios de comunicación se centran en temas como biodiversidad, recursos naturales, y especies en vía de extinción, mientras que en la siguiente década los temas de energía, principalmente la energía nuclear, cobran mucha importancia, y la mirada de la prensa adquiere un carácter de denuncia. En ese entonces la gran mayoría de los periodistas que cubre ambiente, son 'verdes'. En los años ochenta el carácter se torna más profesional, de tal manera que los medios "informan" sobre contaminación atmosférica, acuática, acústica e industrial; sobre disposición de desechos tóxicos y tráfico de especies amenazadas. En los años noventa se hace sentir la reflexión que considera a la sociedad como causa y como objeto de los efectos de los cambios en el ambiente. Los medios informan sobre aspectos demográficos y económicos para el desarrollo sostenible y a partir del año 2000, la información se centra en la biotecnología y en los desastres naturales, en los que se incluye el calentamiento global y el aumento en la destrucción de la capa de ozono. El enfoque periodístico se concentra en acciones por parte de quienes toman desiciones y de organizaciones no gubernamentales, entre otros.

1 Datos tomados de dos conferencias: 1. Evolución y tendencias de la gestión ambiental en America Latina, por Manuel Rodriguez, ex ministro de medio ambiente, en el Seminario Internacional "La politica ambiental en America Latina", Bogotá, nov 6, 2003; y en el Foro Nacional Ambiental, Bogotá, nov 8, 2006. 2. John Bejarano, Fondo Biocomercio, Bogotá, 2006. 
Quizá vale la pena recordar que, en el caso de Colombia, el 12 de julio de 1785 en Santa Fe de Bogotá circula el Aviso del Terremoto, que da cuenta del movimiento que sacudió a la ciudad, en aquella época parte del Nuevo Reino de Granada, como respuesta a la necesidad de los pobladores de entonces de conocer más detalles de lo sucedido. Es la primera publicación periódica (circularon tres números) impresa en Colombia que informa sobre un hecho que no solamente tiene que ver con el tema ambiental, un desastre natural, sino cuyo contenido no es religioso. Informar; ese era el objeto de la publicación, como debe ser hoy en día la intención de las agendas ambientales en los medios de comunicación.

Desde sus primeras manifestaciones, el periodismo ambiental se bate entre el riesgo y la incertidumbre, y sus consecuencias en la salud humana y en la propia naturaleza. ¿Cómo medir la magnitud de los eventos de la naturaleza, la probabilidad de que ocurran, las posibles consecuencias y los efectos que tendrán en las personas que están expuestas?

El periodismo ambiental se confunde a veces con el periodismo en salud y tiene mucho que ver con el periodismo científico, por la seriedad de los temas, y por la incidencia que los acontecimientos de la naturaleza ejercen en el ser humano. De tal manera, que algunos de los grandes aliados de los periodistas que cubren temas ambientales se encuentran en la comunidad científica y de salud, en los epidemiólogos, geólogos, biólogos, botánicos, zoólogos, expertos en clima, ingenieros ambientales, arqueólogos, antropólogos, por mencionar sólo algunos de ellos.

\section{El periodismo ambiental}

Si uno quisiera definir algunas de las funciones del periodismo ambiental, debería partir definitivamente de la necesidad de mantener informada a la ciudadanía sobre estos temas. De la información se deriva otra función que puede cumplir y tiene que ver con apoyar y aumentar la comprensión pública de los temas ambientales y del desarrollo sostenible, promover un periodismo basado en la evidencia más que en la emotividad y asegurar la calidad de la reportería, pues ella influye en la percepción que el ciudadano receptor pueda tener alrededor de estos temas. De una reportería muy profesional y un adecuado trabajo periodístico se desprendería además, que la información puede generar la creación de políticas públicas para el bienestar de la comunidad.

El periodismo ambiental debe ser proactivo, y adelantarse a los hechos; debe ofrecer respuestas y soluciones a los temas que son noticia en el siglo XXI en nuestro país, como la pobreza, la contaminación ambiental, las enfermedades infecciosas, los organismos genéticamente modificados (OGM) y los avances de la biotecnología y la genética, los cultivos ilícitos, entre otros.

Y se debe entender que los temas que le competen se insertan en la búsqueda del bienestar de la población, y por ello, no es posible evadir aspectos políticos, económicos, internacionales y sociales, que la mayoría de las veces, envuelven las historias periodísticas sobre ambiente.

Basta con recordar los Objetivos de Desarrollo del Milenio, ODM, revisarlos detenidamente y entender que del bienestar del ambiente, y de los recursos naturales renovables y no renovables que nos ofrecen, depende en buena medida en cumplir, al menos en parte, con estos derroteros trazados por la Organización de Naciones Unidas, y que sus países miembros deben cumplir al año 2015.

\section{Objetivos de desarrollo del milenio}

1. Erradicar la pobreza extrema y el hambre

2. Lograr la enseñanza primaria universal

3. Promover la igualdad entre los géneros y

la autonomía de la mujer

4. Reducir la mortalidad infantil

5. Mejorar la salud materna

6. Combatir el VIH/SIDA, el paludismo y

otras enfermedades

7. Garantizar la sostenibilidad del medio ambiente

8. Fomentar una asociación mundial para el desarrollo

Un periodista que cubre el tema ambiental debe tener un interés por las disciplinas científicas que se incluyen en las ciencias de la tierra prioritariamente, y estar actualizado en legislación y política ambientales, tanto en lo nacional como en lo internacional. Debe tener capacidad para "transcodificar"2 la información generada

2 "Transcodificar" es un término acuñado por el periodista científico español Manuel Calvo Hernando, que, en líneas generales, significa traducir en un contexto determinado. No se trata pues de una traducción literal, sino adaptada al entorno y al contexto donde se inserta. 
por la comunidad científica y en general, de quienes manejan los temas ambientales, pues no escapan a la utilización de un lenguaje que no necesariamente corresponde al que maneja la ciudadanía en general.

Un periodista ambiental tiene la responsabilidad de buscar la evidencia científica y sustentada como base para la entrega de sus historias en los medios, lo que le evitará convertirse en el vocero de agendas específicas de sus fuentes o del activismo de determinadas corrientes ambientales, o asumir actitudes proteccionistas. Asimismo, debe ser transparente con sus fuentes, y tener la capacidad de involucrar a sus lectores, para que sean ellos quienes lleguen a conclusiones y actúen en consecuencia.

De una investigación exhaustiva, pero enfocada, el periodista evitará caer en posiciones reduccionistas dentro del análisis de los temas, teniendo en cuenta que los temas ambientales exigen ir más allá de la información básica.

La información periodística sobre el tema ambiental deberá tener las siguientes características: un balance que se inicie con la consulta de las diferentes fuentes; plasmar las diferentes posiciones que estén verdaderamente sustentadas; ser precisa, tener rigor técnico; apuntarle a la objetividad, o por lo menos, a la imparcialidad $^{3}$, o a la honestidad ${ }^{4}$, si tenemos en cuenta que la objetividad es un término complejo, difícil de alcanzar; apuntarle también a que sea completa, a que involucre todos los elementos necesarios posibles de conseguir, aunque también es importante dejar una ventana abierta con información no entregada que pueda llevar al desarrollo de otra información sobre el mismo tema luego de un período de tiempo que lo definirá el criterio periodístico; una información útil para el ciudadano, lo que elevará el grado de impacto de la información periodística; y por supuesto, una información que sea verdadera, que se ajuste a la realidad de los hechos o de las posiciones de sus protagonistas, y que sea responsable.
La responsabilidad va de la mano de la ética periodística y cobija no solamente al reportero, sino también al editor, al jefe de redacción y al columnista, incluso al titulador, al ilustrador, y al diagramador. Vale la pena mencionar el código japonés de ética periodística, cuando afirma que: "Para los comentaristas editoriales también el objetivo es la información verídica. Aunque la interpretación de los hechos es subjetiva y susceptible de error, debe fundarse en un conocimiento y una versión verdadera de los hechos" 5 .

\section{La historia ambiental en los medios masivos de comunicación}

Esa historia que perseguimos a diario periodistas y reporteros, va tomando forma desde el momento en que iniciamos el trabajo creativo de generación de ideas. Claro, muchas veces la agenda impone temas, - así es el periodismo- , pero otras tantas es viable y recomendable sugerir historias que son noticia, pero que no son muy visibles en la agenda pública.

Arturo Larena ${ }^{6}$ dice que para encontrar una buena historia se necesita, en primer lugar, buena preparación para identificarla y olfato periodístico. No menos importante, señala, son los criterios de actualidad, novedad, relevancia, proximidad, conflicto, impacto, grado de compromiso y pertinencia.

La generación de ideas surge de un trabajo editorial de equipo, del interés personal, del seguimiento permanente a los diferentes temas, de la construcción de redes de fuentes vivas e institucionales. Para continuar el trabajo periodístico una vez definida la idea de nuestra siguiente historia, la labor no se limita a reportear, escribir y publicar, como muchos piensan. Se necesita tiempo para leer, para consultar, para confrontar, para visitar, para asistir a eventos y conferencias que no necesariamente generan una noticia inmediata, pero que sí generan conocimiento, nos fortalecen como periodistas, y nos convierten en personas mucho más idóneas y más sólidas para ejercer nuestro oficio.

3 Así la denomina el periodista Edgar Maldonado, de Prensa Verde.

4 Así prefiere referirse a ella Arturo Larena Larena, en el Taller de Cobertura de Temas Ambientales organizado por la Fundación Nuevo Periodismo Iberoamericano, del 14 al 17 de noviembre de 2006 en Caracas, Venezuela.

5 María Teresa Herrán y Javier Darío Restrepo (2000). Ética para periodistas. Bogotá: Tercer Mundo Editores. Edición ampliada para Latinoamérica.

6 Relatoría de Maye Primera Garcés, durante el Taller de Cobertura de Temas Ambientales organizado por la Fundación Nuevo Periodismo Iberoamericano. Caracas, Venezuela, 14 al 17 de noviembre de 2006. 
Desafortunadamente no es fácil convencer a nuestras casas editoriales de la importancia de disponer de ese tiempo y de ese espacio para enriquecer el conocimiento, lo que se reflejaría a mediano y largo plazo, no necesariamente en la inmediatez de nuestro oficio. Tiempo y espacio para poder plasmar en nuestras historias periodísticas un conocimiento mucho más fundamentado y justificado, con criterio, que nos fortalezca la capacidad de evaluar situaciones y fuentes, condiciones mínimas para un producto de calidad.

Las fuentes son el pilar de nuestras historias, y la base de la etapa de reportería e investigación periodística. Si consultamos las fuentes más apropiadas podríamos garantizar una historia periodística de impacto. ¿Cómo sabemos cuáles son las fuentes más apropiadas? Lo aprendemos con el tiempo, con la experiencia, con la constancia, con el conocimiento que vamos adquiriendo. Lo aprendemos cuando entendemos que hay conflictos de interés en los testimonios, que en la ciencia hay cabida para la incertidumbre y que no es exacta, que los hallazgos y los resultados científicos pueden tener varias lecturas, que hay conflicto también entre los avances científicos y tecnológicos y los valores sociales, económicos, morales o políticos.

Un primer listado de las posibles fuentes vivas en periodismo científico incluye a los investigadores en universidades, centros de investigación (sociólogos, biólogos, químicos, ecólogos, agrónomos, climatólogos, arquitectos e ingenieros, economistas, etcétera), los funcionarios de organizaciones ambientales, de organizaciones oficiales nacionales e internacionales, los tomadores de decisiones, los voceros de la industria, quienes definen las legislaciones y los ciudadanos.

En el listado de fuentes institucionales se incluyen revistas científicas, especializadas, portales de las instituciones, boletines de prensa, instrumentos de visibilidad de los diferentes organismos, libros, películas, congresos, reuniones de expertos, documentos de política, en fin, nos haríamos interminables enumerándolos a todos.

Pero más que enumeración de fuentes, lo importante es aprender a consultar aquellas fuentes más idóneas, más confiables, más veraces, lo que, como decíamos anteriormente, es un primer paso para garantizar el éxito de nuestros artículos.

Más aún, buscar en lo nacional para luego escudriñar en lo internacional es una estrategia que permite hacer visible la ciencia y sus voceros colombianos. Por ejemplo, el Instituto Colombiano para el Desarrollo de la Ciencia y la Tecnología Francisco José de Caldas, Colciencias, tiene un instrumento de búsqueda útil para los periodistas. Se trata de la plataforma Scienti, donde el navegante (www.colciencias.gov.co) puede encontrar las hojas de vida de todos los miembros de la comunidad científica colombiana que forman parte del desarrollo científico y tecnológico del país, de los grupos de investigación avalados por el Instituto por su calidad, e información sobre sus trabajos, sus líneas de acción, los proyectos que adelantan, entre otra información clave para el periodista.

Otras páginas claves para consulta de un periodista ambiental son:

\begin{tabular}{|l|l|}
\hline \multicolumn{1}{|c|}{ INSTITUCIÓN } & \multicolumn{1}{|c|}{ PÁGINA } \\
\hline $\begin{array}{l}\text { Ministerio de Ambiente, Vivien- } \\
\text { da y Desarrollo Territorial }\end{array}$ & www.minambiente.gov.co \\
\hline $\begin{array}{l}\text { Instituto de Investigaciones } \\
\text { Marinas y Costeras José Benito } \\
\text { Vives de Andreis }\end{array}$ & www.invemar.org.co \\
\hline $\begin{array}{l}\text { Instituto de Hidrología, Meteo- } \\
\text { rología y Estudios Ambientales }\end{array}$ & www.ideam.gov.co \\
\hline $\begin{array}{l}\text { Instituto de Investigación de } \\
\text { Recursos Biológicos Alexander } \\
\text { Von Humboldt }\end{array}$ & www.humboldt.org.co \\
\hline $\begin{array}{l}\text { Corporación Autónoma Regio- } \\
\text { nal de los Valles del Sinú y del } \\
\text { San Jorge }\end{array}$ & www.cvs.gov.co \\
\hline $\begin{array}{l}\text { Instituto Colombiano para el } \\
\text { Desarrollo de la Ciencia y la } \\
\text { Tecnología Francisco José de } \\
\text { Caldas, Colciencias }\end{array}$ & www.colciencias.gov.co \\
\hline $\begin{array}{l}\text { Asociación Colombiana para el } \\
\text { Avance de la Ciencia }\end{array}$ & www.acac.org.co \\
\hline $\begin{array}{l}\text { Academia Colombiana de Cien- } \\
\text { cias Exactas, Físicas y Naturales }\end{array}$ & www.accefyn.org.co \\
\hline Universia & www.universia.net.co \\
\hline $\begin{array}{l}\text { Observatorio Colombiano de } \\
\text { Ciencia y Tecnología }\end{array}$ & www.ocyt.org.co \\
\hline
\end{tabular}


Universidades nacionales:

\begin{tabular}{|l|l|}
\hline \multicolumn{1}{|c|}{ INSTITUCIÓN } & \multicolumn{1}{c|}{ PÁGINA } \\
\hline Universidad de los Andes & www.uniandes.edu.co \\
\hline $\begin{array}{l}\text { Universidad Nacional } \\
\text { de Colombia }\end{array}$ & www.unal.edu.co \\
\hline Universidad de Antioquia & www.udea.edu.co \\
\hline $\begin{array}{l}\text { Universidad Industrial } \\
\text { de Santander }\end{array}$ & www.uis.edu.co \\
\hline Universidad de Cartagena & www.unicartagena.edu.co \\
\hline Universidad del Valle & www.univalle.edu.co \\
\hline
\end{tabular}

Entre los portales internacionales que vale la pena tener en cuenta están:

\begin{tabular}{|l|l|}
\hline \multicolumn{1}{|c|}{ INSTITUCIÓN } & \multicolumn{1}{c|}{ PÁGINA } \\
\hline Red de Ciencia y Desarrollo & www.scidev.net \\
\hline $\begin{array}{l}\text { Asociación Americana para } \\
\text { el Avance de la Ciencia }\end{array}$ & www.eurekalert.com \\
\hline $\begin{array}{l}\text { Federación Mundial de } \\
\text { Periodistas Científicos }\end{array}$ & www.wfsj.com \\
\hline $\begin{array}{l}\text { Federación Internacional de } \\
\text { Periodistas Ambientales }\end{array}$ & www.ifej.org \\
\hline $\begin{array}{l}\text { National Association of } \\
\text { Science Writers }\end{array}$ & www.nasw.org \\
\hline $\begin{array}{l}\text { Public Communication of } \\
\text { Science and Technology } \\
\text { Network }\end{array}$ & www.pcstnetwork.org \\
\hline $\begin{array}{l}\text { Society of Environmental } \\
\text { Journalism }\end{array}$ & www.sej.org \\
\hline $\begin{array}{l}\text { Revista electronica de } \\
\text { ecología y medio ambiente }\end{array}$ & www.infoecologia.com \\
\hline
\end{tabular}

La tarea del periodista ambiental incluye la búsqueda permanente de nuevas voces autorizadas y sólidas, y nutrirse con información de las diferentes partes involucradas en el tema ambiental, utilizando al máximo su sabiduría y experiencia al seleccionar, utilizar o descartar información que recibe cotidianamente.

\section{Córdoba, ambiente, ciencia y periodismo}

Unas pinceladas sobre la investigación científica y ambiental en Colombia y en Córdoba, permitió a los participantes en el Diplomado de Periodismo Ambiental realizado en Montería tener una idea general sobre la situación de su departamento, en un contexto nacional.*

De acuerdo con datos del Observatorio Colombiano de Ciencia y Tecnología, OCyT, el gasto nacional en ciencia y tecnología con relación al Producto Interno Bruto, PIB, en el 2004 estuvo entre el $0.179 \%$ y el $0.49 \%$.

La inversión de Colciencias en 2004 fue de Col\$87.371'220.000. El Gobierno nacional ha anunciado, que para el año 2008 , será de 160 mil millones aproximadamente.

El gasto en ciencia y tecnología por institución al 2004 fue así:

\begin{tabular}{|l|c|}
\hline Instituciones públicas & $45.03 \%$ \\
\hline Empresas & $5.77 \%$ \\
\hline $\begin{array}{l}\text { Instituciones de educación } \\
\text { superior }\end{array}$ & $36.52 \%$ \\
\hline $\begin{array}{l}\text { Centros investigación } \\
\text { privados }\end{array}$ & $12.68 \%$ \\
\hline
\end{tabular}

Los grupos de investigación activos ${ }^{8}$ en el país a enero del 2005 eran 2.244, de los cuales, 187 estaban catalogados con trabajos de ciencias del medio ambiente y el hábitat. El total de grupos registrados era de 3.277 , de los cuales, 276 trabajan en ciencias del medio ambiente y el hábitat.

A enero del 2005, el departamento de Córdoba tenía 21 grupos de investigación activos y 38 registrados. Asimismo, los investigadores activos de Córdoba sumaban 142 de 13.036 y 193 registrados, de 15.655 .

Finalmente, a enero de 2005, Córdoba realizaba 167 proyectos de investigación en cuatro áreas de investigación, así:

\footnotetext{
* Convenio 140 CVS - Red Colombiana de Formación Ambiental, abril - mayo, 2007.

7 Estimación del gasto en CyT con un alto nivel de incertidumbre, de acuerdo con OCyT. Colombia 2005: Indicadores de ciencia y tecnología. Observatorio Colombiano de Ciencia y Tecnología. Bogotá: Colciencias, 2006.

8 Significa que tenían un proyecto activo en el momento de la medición.
} 


\begin{tabular}{|l|c|}
\hline Agrícolas & 60 \\
\hline Sociales y Humanas & 59 \\
\hline Médicas & 25 \\
\hline Naturales y exactas & 23 \\
\hline TOTAL & 167 \\
\hline
\end{tabular}

ma Regional de los Valles del Sinú y del San Jorge, CVS, a 21 periodistas de prensa escrita, radio y televisión de la región, quienes respondieron identificaron ocho temas diferentes sobre el medio ambiente y su relación con los aspectos biológicos, sociales, políticos, económicos y jurídicos de la región: manejo de humedales; manejo de residuos sólidos; erosión marina frente a turismo; biodiversidad; contaminación auditiva; uso

En el momento de escribir este artículo, Colciencias evaluaba 1.555 anteproyectos de investigación que presentó la comunidad científica y tecnológica nacional a la convocatoria para su posible cofinanciación en el 2007, en la modalidad de "recuperación contingente". Quiero resaltar el puesto que ocupó la oferta en investigación sobre medio ambiente, lo que da cuenta del interés de la comunidad científica nacional por generar conocimiento en estos temas. Así fue la propuesta de los científicos:

\begin{tabular}{|l|c|}
\hline Ciencias básicas & $23 \%$ \\
\hline Ciencias sociales y humanas & $18 \%$ \\
\hline Medio ambiente y hábitat & $11 \%$ \\
\hline Ciencias agropecuarias & $10 \%$ \\
\hline Desarrollo industrial & $9 \%$ \\
\hline Educación & $8 \%$ \\
\hline Biotecnología & $7 \%$ \\
\hline Energía y minería & $6 \%$ \\
\hline $\begin{array}{l}\text { Electrónica, telecomunica- } \\
\text { ciones e informática }\end{array}$ & $5 \%$ \\
\hline $\begin{array}{l}\text { Ciencia y tecnología del } \\
\text { mar }\end{array}$ & $3 \%$ \\
\hline
\end{tabular}

Mientras tanto la Universidad Nacional de Colombia informó que seleccionó un total de 354 proyectos en ciencia, tecnología, arte e innovación en sus siete sedes, para financiarlos con un total de ocho mil millones de pesos. Algunas de las propuestas ganadoras buscan desentrañar principios bioactivos de plantas medicinales, estudiar y aprovechar los productos marinos y las frutas de Colombia; crear un observatorio sobre infancia; producir combustibles alternativos; expresar plásticamente una obra literaria o dar cuenta de las migraciones de colombianos hacia el Ecuador ${ }^{9}$.

En una encuesta reallizadla por la Conpronaccióm Auttónm-

Los problemas ambientales del departamento, en un momento en que el desbordamiento del río Sinú deja miles de damnificados ${ }^{10}$, pone en evidencia la necesidad urgente de que los medios masivos de comunicación regionales y nacionales fortalezcan su capacidad periodística para informar responsablemente sobre los hechos sucedidos y llama la atención de la ciudadanía sobre las causas y las consecuencias de las tragedias naturales, en las que, muchas veces, la acción del hombre tiene que ver con las causas, y la tragedia humana es el centro de atención de las consecuencias.

Según informaron los participantes y el encargado de la página de medio ambiente en El Meridiano de Córdoba, durante el módulo que estuvo a mi cargo, "Periodismo ambiental y sociedad del conocimiento", desde este año de 2007, dicho diario publica una página mensual, que circula un lunes de cada mes.

El ambiente es un tema transversal y estos acontecimientos se convierten en prueba de fuego para definir si es posible que el periodismo sea una herramienta que contribuya al desarrollo y al avance intelectual de nuestras sociedades cuando se entrega una información confiable.

En las historias periodísticas, que se inician desde la generación de la idea, pasan por la reportería o investigación periodística, continúan por la organización de los datos acopiados y la redacción de la historia, pero también, e igualmente importante, siguen los procesos de edición y corrección, y luego la publicación, el impacto en el receptor es cada vez más importante.

9 Boletines de prensa de Colciencias y de la Universidad Nacional de Colombia. Mayo y junio de 2007.

$10 \mathrm{Al}$ momento de escribir este artículo y en pleno desarrollo de las actividades del Diplomado, el desbordamiento del Río Sinú y sus consecuencias se convirtieron en noticia de primera página de los diferentes medios de comunicación nacionales. 
¿Cómo medir el impacto? Las investigaciones al respecto en el campo de la ciencia, la tecnología, el ambiente y la salud, se han iniciado recientemente, aunque hay experiencias interesantes, son aún muy específicas y por tanto, es difícil adaptarlas a otros contextos y a otras latitudes. En una primera aproximación, el periodista, el editor, el medio de comunicación deberá intentar responder a las siguientes preguntas: El artículo publicado... ¿generó opinión? ¿generó actitudes? ¿generó otro tipo de reacción?

El periodismo, prioritariamente, tiene el objetivo de informar. Sin embargo, la entrega de nuevo conocimiento genera más que información: María Pilar Diezhandino, experta en periodismo de servicio dice que este tipo de periodismo "busca un cambio de conducta individual, personal y luego un cambio de conducta sociopolítica. Si es informativo exclusivamente no es de servicio" 11 .

Yo no me atrevería a decir que el periodismo ambiental es un periodismo de servicio. Pero sí quisiera compartir con el lector la siguiente idea del Programa de las Naciones Unidas para el Medio Ambiente, PNUMA:

Para hacer frente a los problemas ambientales es fundamental que los medios de comunicación dispongan de mejor información sobre la materia y después que la transmitan adecuadamente al público. El éxito de la comunicación ambiental no ha de medirse por la aceptación pública de las soluciones formuladas por los encargados de tomar decisiones; se alcanza cuando un público bien informado escoge las mejores soluciones con conocimiento de causa. Una de las funciones más importantes de los medios de comunicación es ampliar el público que participa en el debate sobre una cuestión concreta ${ }^{12}$.

Teniendo en cuenta que el tema ambiental es uno de los que más interesa al mundo entero, de acuerdo con estudios realizados por la National Science Foundation para Estados Unidos, el Eurobarómetro para Europa, Andiarios y El Tiempo, en Colombia, quiero terminar con una frase de mi mentor y maestro en periodismo científico, el periodista español Manuel Calvo Hernando: "Nuestros artículos deben tener un balance entre un optimismo prudente y un pesimismo esperanzador".

\section{Bibliografía}

- Observatorio Colombiano de Ciencia y Tecnología. Colombia 2005: Indicadores de ciencia y tecnología. Bogotá: Colciencias, 2006. p. 298.

- $\quad$ Medio ambiente, medios y academia, Universidad Nacional de Colombia. Bogotá: 1993.

- CALVO HERNANDO, Manuel (1997). Manual de periodismo científico. Barcelona: Bosch Comunicaciones. p. 242.

- DIEZHANDINO, Ma. Pilar (1994). Periodismo de servicio. Barcelona: Bosch Comunicaciones. p. 214.

- ESTEVE RAMÍREZ, Francisco y FERNÁNDEZ DEL MORAL, Javier (1999). Áreas de especialización periodística. Madrid: Fragua. p. 330.

- HERRÁN, María Teresa y RESTREPO, Javier Darío (2000), Ética para periodistas. Edición ampliada para Latinoamérica. Bogotá: Tercer Mundo Editores. p. 361

- NELSON, Peter. Ten practical tips for environmental reporting. The Center for Foreign Journalists y World Wide Fund for Nature. 1995.

- PRENAFETA JENKIN, Sergio (2002). Teoría y práctica del periodismo científico. Santiago de Chile: Editorial Andrés Bello. p. 304.

- WEST, Bernadette, SANDMAN, Peter M, GREENBERG, Michael R. (1994). The reporter's environmental handbook. New Jersey: Rutgers University Press. p.346. 